Discrete Comput Geom 33:125-142 (2005)

DOI: $10.1007 / \mathrm{s} 00454-004-1109-3$

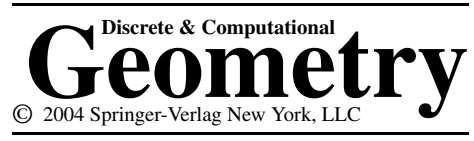

\title{
An Identity Relating Moments of Functionals of Convex Hulls
}

\author{
Christian Buchta \\ Department of Mathematics, Salzburg University, \\ Hellbrunner Strasse 34, A-5020 Salzburg, Austria \\ christian.buchta@sbg.ac.at
}

\begin{abstract}
Denote by $K_{n}$ the convex hull of $n$ independent random points distributed uniformly in a convex body $K$ in $\mathbb{R}^{d}$, by $V_{n}$ the volume of $K_{n}$, by $D_{n}$ the volume of $K \backslash K_{n}$, and by $N_{n}$ the number of vertices of $K_{n}$. A well-known identity due to Efron relates the expected volume $E D_{n}$ - and thus $E V_{n}$ - to the expected number $E N_{n+1}$. This identity is extended from expected values to higher moments.

The planar case of the arising identity for the variances provides in a simple way the corrected version of a central limit theorem for $D_{n}$ by Cabo and Groeneboom ( $K$ being a convex polygon) and an improvement of a central limit theorem for $D_{n}$ by Hsing ( $K$ being a circular disk). Estimates of $\operatorname{var} D_{n}$ ( $K$ being a two-dimensional smooth convex body) and $\operatorname{var} N_{n}$ ( $K$ being a $d$-dimensional smooth convex body, $d \geq 4$ ) are obtained.

The identity for moments of arbitrary order shows that the distribution of $N_{n}$ determines $E V_{n-1}, E V_{n-2}^{2}, \ldots, E V_{d+1}^{n-d-1}$. Reversely it is proved that these $n-d-1$ moments determine the distribution of $N_{n}$ entirely. The resulting formula for the probability that $N_{n}=k(k=$ $d+1, \ldots, n)$ appears to be new for $k \geq d+2$ and yields an answer to a question raised by Baryshnikov. For $k=d+1$ the formula reduces to an identity which has been repeatedly pointed out.
\end{abstract}

\section{Introduction and Main Results}

Write $\mathcal{K}^{d}$ for the set of all convex bodies (convex compact sets with non-empty interiors) in $\mathbb{R}^{d}$. Fix $K \in \mathcal{K}^{d}$, and choose points $x_{1}, \ldots, x_{n} \in K$ randomly, independently, and according to the uniform distribution on $K$. The set $K_{n}=\operatorname{conv}\left\{x_{1}, \ldots, x_{n}\right\}$ is a random polytope. Denote the volume of $K_{n}$ by the random variable $V_{n}$, the volume of $K \backslash K_{n}$ by the random variable $D_{n}$, and the number of vertices of $K_{n}$ by the random variable $N_{n}$.

Numerous papers were devoted to the question of how to determine the expected values of these and similar random variables (e.g. the surface area or the number of facets of $K_{n}$ ) for certain classes of convex bodies $K$. Particular attention was paid to the asymptotic behaviour of the expected values as $n$ tends to infinity. It is beyond the scope 
of this paper to list the results on expected values. Surveys were given by Affentranger [1], Buchta [10], Gruber [19], Schneider [39], [40], and Weil and Wieacker [43]. Many references are also contained in [5], [13], and [14].

In contrast to the large number of results on expected values, not much is known so far about variances and higher moments. The available results concern throughout very special convex bodies $K$ and either just $n=d+1$ random points (spanning a random simplex in $K$ ) or the asymptotic behaviour as $n$ tends to infinity. (In the case of $n<d+1$ random points trivially $V_{n}=0, D_{n}=\operatorname{vol} K$, and $N_{n}=n$ with probability one.)

The distribution function (implying all moments) of $V_{n}$ has apparently only been derived in the case $d=2$ and $n=3$ : by Alagar [2] if $K$ is a triangle and by Henze [20] if $K$ is a circular disk or a parallelogram. All moments of $V_{n}$ in the case $d=2$ and $n=3$ had been obtained before by Miles [31] for the circular disk and by Reed [33] for the triangle and the parallelogram. The result derived by Miles is much more general and comprises all moments of $V_{d+1}$ for the $d$-dimensional ball. Reed also obtained the second moment of $V_{d+1}$ if $K$ is a $d$-dimensional simplex; this result was supplemented in the three-dimensional case by Mannion [27] who calculated the fourth and the sixth moment of $V_{4}$ if $K$ is a tetrahedron.

Whereas all these results concern $n=d+1$ random points, substantial progress in regard of the asymptotic behaviour as $n$ tends to infinity was achieved by deriving the following central limit theorems. The first two results concern the random variable $N_{n}$ and are due to Groeneboom [18]: If $K$ is a convex polygon with $r$ vertices, then

$$
\frac{N_{n}-\frac{2}{3} r \log n}{\sqrt{\frac{10}{27} r \log n}} \stackrel{\mathcal{D}}{\rightarrow} \mathcal{N}(0,1)
$$

as $n \rightarrow \infty$, where $\stackrel{\mathcal{D}}{\rightarrow}$ denotes convergence in distribution and $\mathcal{N}(0,1)$ is the standard normal distribution. If $K$ is a circular disk, then

$$
\frac{N_{n}-2 \pi c_{1} n^{1 / 3}}{\sqrt{2 \pi c_{2} n^{1 / 3}}} \stackrel{\mathcal{D}}{\rightarrow} \mathcal{N}(0,1),
$$

with $c_{1}=(2 / 3 \pi)^{1 / 3} \Gamma\left(\frac{5}{3}\right) \approx 0.53846$ and $c_{2}$ given by an integral which was evaluated numerically; see also Section 5.3. Subsequently, Cabo and Groeneboom [15] considered the random variable $D_{n}$ in the case that $K$ is a convex polygon with $r$ vertices and stated that

$$
\frac{D_{n}-\frac{2}{3} r n^{-1} \log n}{\sqrt{\frac{100}{189} r n^{-1} \log n}} \stackrel{\mathcal{D}}{\rightarrow} \mathcal{N}(0,1) .
$$

In the case that $K$ is a circular disk, Hsing [21] proved that

$$
\lim _{n \rightarrow \infty} \operatorname{var} D_{n} n^{5 / 3}=\sigma^{2},
$$

with a number $\sigma^{2}<\infty$, and that

$$
\left(D_{n}-E D_{n}\right) n^{5 / 6} \stackrel{\mathcal{D}}{\rightarrow} \mathcal{N}\left(0, \sigma^{2}\right) .
$$


The number $\sigma^{2}$ is given in terms of an integral such that the integrand is the covariance of certain random variables. It is not obvious how to evaluate the integral or at least to conclude whether $\sigma^{2}>0$. A result in higher dimensions similar to (1.4) is due to Küfer [26], who showed that $\operatorname{var} D_{n}$ is at most of order $n^{-(d+3) /(d+1)}$ if $K$ is a $d$-dimensional ball.

The results for circular disks and balls carry with them the respective results for ellipses and ellipsoids, since $N_{n}$, (vol $\left.K\right)^{-1} D_{n}$, and (vol $\left.K\right)^{-1} V_{n}$ are invariant under non-singular affine transformations of $K$.

As for the results on expected values, the simple identity due to Efron [17],

$$
\frac{E D_{n}}{\operatorname{vol} K}=\frac{E N_{n+1}}{n+1}
$$

or, equivalently,

$$
\frac{E V_{n}}{\operatorname{vol} K}=1-\frac{E N_{n+1}}{n+1},
$$

turned out to be a very useful tool, see, e.g., p. 42 of [15]. Hence the question arises whether similar identities also exist for variances and higher moments. In particular: Given $n \in \mathbb{N}$, is there an $m \in \mathbb{N}$ such that $\operatorname{var} N_{m}$ on the one hand and $\operatorname{var} D_{n} \operatorname{or} \operatorname{var} V_{n}$ on the other hand are related by an identity?

In the following we give affirmative answers to these questions. In Section 2 we prove that, for every $K \in \mathcal{K}^{d}$, every $n \in \mathbb{N}$, and every $k \in \mathbb{N}$,

$$
\frac{E V_{n}^{k}}{(\operatorname{vol} K)^{k}}=E \prod_{i=1}^{k}\left(1-\frac{N_{n+k}}{n+i}\right)
$$

and, consequently,

$$
\frac{E D_{n}^{k}}{(\operatorname{vol} K)^{k}}=\sum_{j=1}^{k}(-1)^{j-1}\left(\begin{array}{l}
k \\
j
\end{array}\right) E\left(1-\prod_{i=1}^{j}\left(1-\frac{N_{n+j}}{n+i}\right)\right) .
$$

Thus the $k$ th moment of $V_{n}$ can be expressed by the first $k$ moments of $N_{n+k}$ :

$$
\begin{aligned}
\frac{E V_{n}^{k}}{(\operatorname{vol} K)^{k}}= & 1-\left(\frac{1}{n+1}+\cdots+\frac{1}{n+k}\right) E N_{n+k} \\
& +\cdots+(-1)^{k} \frac{1}{(n+1) \cdots(n+k)} E N_{n+k}^{k},
\end{aligned}
$$

and, analogously, the $k$ th moment of $D_{n}$ is a linear function of the first moment of $N_{n+1}$, the first two moments of $N_{n+2}, \ldots$, and the first $k$ moments of $N_{n+k}$.

As an immediate consequence of (1.8) and (1.9), which we consider in Section 3, we obtain that

$$
\frac{\operatorname{var} V_{n}}{(\operatorname{vol} K)^{2}}=\frac{\operatorname{var} D_{n}}{(\operatorname{vol} K)^{2}}=\frac{\operatorname{var} N_{n+2}+d_{n+2}}{(n+1)(n+2)},
$$

where $d_{n+2}$ is entirely determined by the expected values $E N_{n+1}$ and $E N_{n+2}$. (See Corollary 1 in Section 3 for the precise definition of $d_{n}$; note also Remark 4.) 
If $K$ is a convex polygon with $r$ vertices, a classical result due to Rényi and Sulanke [37] says that $E N_{n}=\frac{2}{3} r \log n+c_{0}(K)+o(1)$ as $n \rightarrow \infty$ (where the constant $c_{0}(K)$ depends on the shape of $K$ ). A more detailed investigation by Buchta and Reitzner [13] shows that $o(1)$ can be replaced by $O\left(n^{-1}\right)$. Then it is immediately seen that $d_{n} \sim \frac{2}{3} r \log n$. According to Groeneboom [18, p. 328], it can be deduced from the central limit theorem stated as (1.1) in the present paper that $\operatorname{var} N_{n} \sim \frac{10}{27} r \log n$ as $n \rightarrow \infty$. (See the comment on this conclusion in Section 5.4.) Consequently the identity (1.10) yields $(\operatorname{vol} K)^{-2} \operatorname{var} D_{n} \sim \frac{28}{27} r n^{-2} \log n$. This is incompatible with the corresponding conclusion for the variance of $D_{n}$ from the central limit theorem stated as (1.3) in the present paper and suggests that (1.3) has to be replaced by

$$
\frac{(\operatorname{vol} K)^{-1} D_{n}-\frac{2}{3} r n^{-1} \log n}{\sqrt{\frac{28}{27} r n^{-2} \log n}} \stackrel{\mathcal{D}}{\rightarrow} \mathcal{N}(0,1) .
$$

Analogously, if $K$ is a circular disk, a particular case of a further result by Rényi and Sulanke [37] says that $E N_{n} \sim 2 \pi c_{1} n^{1 / 3}$ as $n \rightarrow \infty$, where the constant $c_{1}$ is the same as in (1.2). A refinement of this result due to Gruber [19] and Reitzner [34] exhibits the further terms in the asymptotic expansion of $E N_{n}$ and implies $d_{n} \sim \frac{2}{3} \pi c_{1} n^{1 / 3}$. According to Groeneboom (ibid.), it can likewise be deduced from the central limit theorem stated as (1.2) in the present paper that $\operatorname{var} N_{n} \sim 2 \pi c_{2} n^{1 / 3}$ as $n \rightarrow \infty$. Consequently it follows that $(\operatorname{vol} K)^{-2} \operatorname{var} D_{n} \sim 2 \pi\left(\frac{1}{3} c_{1}+c_{2}\right) n^{-5 / 3}$, and the number occurring in (1.4) and (1.5) is given by

$$
\sigma^{2}=2 \pi\left(\frac{1}{3} c_{1}+c_{2}\right)(\operatorname{vol} K)^{2}>0 .
$$

Furthermore, according to (1.6), $E N_{n} \sim 2 \pi c_{1} n^{1 / 3}$ implies (vol $\left.K\right)^{-1} E D_{n} \sim 2 \pi c_{1} n^{-2 / 3}$ (alternatively, see [38]), and Hsing's result (1.5) can be improved to

$$
\frac{(\operatorname{vol} K)^{-1} D_{n}-2 \pi c_{1} n^{-2 / 3}}{\sqrt{2 \pi\left(\frac{1}{3} c_{1}+c_{2}\right) n^{-5 / 3}}} \stackrel{\mathcal{D}}{\rightarrow} \mathcal{N}(0,1),
$$

where the constants $c_{1}$ and $c_{2}$ are the same as in (1.2).

Beyond that, the simple identity (1.10) gives rise to estimates of $\operatorname{var} D_{n}$ and $\operatorname{var} N_{n}$, respectively, for much more general convex bodies: If $K$ is a $d$-dimensional convex body with sufficiently smooth boundary, a result by Bárány [3] shows that $E N_{n} \sim$ $C(K) n^{(d-1) /(d+1)}$ as $n \rightarrow \infty$, where $C(K)>0$ is known explicitly and depends on the curvature of the boundary. Reitzner [36] obtained further terms in the asymptotic expansion of $E N_{n}$. Hence it follows that $d_{n} \sim((3-d) /(d+1)) C(K) n^{(d-1) /(d+1)}$. Thus $\operatorname{var} N_{n} \geq 0$ implies for $d=2$ that, asymptotically,

$$
\frac{\operatorname{var} D_{n}}{(\operatorname{vol} K)^{2}} \geq \frac{1}{3} C(K) n^{-5 / 3}
$$

and from $\operatorname{var} D_{n} \geq 0$ it follows for $d \geq 4$ that, asymptotically,

$$
\operatorname{var} N_{n} \geq \frac{d-3}{d+1} C(K) n^{(d-1) /(d+1)} .
$$


Presumably, the right-hand sides of (1.11) and (1.12), respectively, give the exact order of the estimated variances and the constants are not "too far" from the exact ones. A similar reasoning is possible for other convex bodies as far as asymptotic expansions of $E N_{n}$ are available.

In Section 4 we return to the general identity (1.8). The fact that $E V_{n}^{k}$ is determined by moments of $N_{n+k}$ trivially implies that $E V_{n}^{k}$ is determined by the distribution of $N_{n+k}$. The argument is the same for any $n$ and any $k$; hence any moment $E V_{n}^{k}$ with $n+k=m$ is determined by the distribution of $N_{m}$. Reversely we derive that, for every $m \in \mathbb{N}$, the distribution of $N_{m}$ is determined by the moments $E V_{j}^{m-j}(j=1, \ldots, m)$. The probabilities $p_{l}^{(m)}$ that $N_{m}=l(l=1, \ldots, m)$ turn out to be

$$
p_{l}^{(m)}=(-1)^{l}\left(\begin{array}{c}
m \\
l
\end{array}\right) \sum_{j=1}^{l}(-1)^{j}\left(\begin{array}{l}
l \\
j
\end{array}\right) \frac{E V_{j}^{m-j}}{(\operatorname{vol} K)^{m-j}} .
$$

This formula—as well as Efron's identity and its generalization-does not depend on the dimension $d$ and is purely combinatorial, indicating that it holds under more general assumptions; see Remark 2. The dimension comes into play if we omit the moments $E V_{1}^{m-1}, \ldots, E V_{d}^{m-d}$, which are zero. Then the summation on the right-hand side of (1.13) starts with $j=d+1$, and the sum is empty if $l \leq d$, corresponding to $p_{1}^{(m)}=\cdots=$ $p_{d}^{(m)}=0$.

Apparently, only the particularly simple case $l=d+1$ of (1.13), where the sum reduces to one summand, has been noticed before. (The expression in this case,

$$
p_{d+1}^{(m)}=\left(\begin{array}{c}
m \\
d+1
\end{array}\right) \frac{E V_{d+1}^{m-d-1}}{(\operatorname{vol} K)^{m-d-1}},
$$

is stated, e.g., in the Handbook of Convex Geometry [43, p. 1396], in the Handbook of Discrete and Computational Geometry [40, p. 169] and in Schneider's survey [39, p. 219].) The probability $p_{m}^{(m)}$, i.e. the probability that $m$ random points are in convex position, was recently investigated by Valtr [41], [42] and Bárány [4].

Related work is due to Baryshnikov [7], Bräker and Hsing [8], Devroye [16], Hueter [22], [23], Jewell and Romano [25, p. 424], Massé [28]-[30], Nagaev and Khamdamov [32], and Reitzner [35].

\section{Extension of Efron's Identity to Higher Moments}

Theorem 1. Let $K \in \mathcal{K}^{d}, n \in \mathbb{N}$, and $k \in \mathbb{N}$. Then

$$
\frac{E V_{n}^{k}}{(\operatorname{vol} K)^{k}}=E \prod_{i=1}^{k}\left(1-\frac{N_{n+k}}{n+i}\right)
$$

and, consequently,

$$
\frac{E D_{n}^{k}}{(\operatorname{vol} K)^{k}}=\sum_{j=1}^{k}(-1)^{j-1}\left(\begin{array}{l}
k \\
j
\end{array}\right) E\left(1-\prod_{i=1}^{j}\left(1-\frac{N_{n+j}}{n+i}\right)\right) .
$$


Proof. Assuming that $n+k$ points are distributed independently and uniformly in $K$, we investigate the number $P_{n, k}$ of subsets of the set of the $n+k$ points which are of the following type: The subset consists of exactly $k$ points, and these $k$ points are contained in the convex hull of the remaining $n$ points. As we are going to express the expected value of the random variable $P_{n, k}$ in two different ways, the first of the claimed identities follows.

We start by considering any fixed arrangement $\left\{x_{1}, \ldots, x_{n+k}\right\}$ of $n+k$ pairwise different points in $K$. Denote by $N_{n+k}\left(x_{1}, \ldots, x_{n+k}\right)$ the number of vertices of $\operatorname{conv}\left\{x_{1}, \ldots, x_{n+k}\right\}$. The number $P_{n, k}\left(x_{1}, \ldots, x_{n+k}\right)$ of subsets of $\left\{x_{1}, \ldots, x_{n+k}\right\}$ consisting of exactly $k$ points which are contained in the convex hull of the remaining $n$ points equals the number of possibilities of choosing $k$ points from those of the $n+k$ points which are not vertices of $\operatorname{conv}\left\{x_{1}, \ldots, x_{n+k}\right\}$. Since, by definition, there are $n+k-N_{n+k}\left(x_{1}, \ldots, x_{n+k}\right)$ non-vertices,

$$
P_{n, k}\left(x_{1}, \ldots, x_{n+k}\right)=\left(\begin{array}{c}
n+k-N_{n+k}\left(x_{1}, \ldots, x_{n+k}\right) \\
k
\end{array}\right) .
$$

Hence the random variables $P_{n, k}$ and $N_{n+k}$ are related by

$$
P_{n, k}=\left(\begin{array}{c}
n+k-N_{n+k} \\
k
\end{array}\right) \text {. }
$$

In particular,

$$
E P_{n, k}=E\left(\begin{array}{c}
n+k-N_{n+k} \\
k
\end{array}\right) .
$$

We now return to a fixed arrangement $\left\{x_{1}, \ldots, x_{n+k}\right\}$ of $n+k$ pairwise different points in $K$. The number $P_{n, k}\left(x_{1}, \ldots, x_{n+k}\right)$ can alternatively be determined in the following way: Check for each of the $\left(\begin{array}{c}n+k \\ k\end{array}\right)$ selections of $k$ points whether the selected points are contained in the convex hull of the remaining $n$ points. As the points are identically distributed, every selection is with the same probability contained in the convex hull of the remaining points. Denote by $p_{n, k}$ this probability, i.e. the probability that $k$ points distributed independently and uniformly in $K$ are contained in the convex hull of $n$ further points distributed independently and uniformly in $K$. Then

$$
E P_{n, k}=\left(\begin{array}{c}
n+k \\
k
\end{array}\right) p_{n, k} .
$$

Finally, we derive an expression for $p_{n, k}$ which is suitable for our purpose. For every fixed arrangement $\left\{x_{1}, \ldots, x_{n}\right\}$ of $n$ points the probability that a point chosen uniformly at random in $K$ falls within $\operatorname{conv}\left\{x_{1}, \ldots, x_{n}\right\}$ is given by $(\operatorname{vol} K)^{-1}$ vol $\operatorname{conv}\left\{x_{1}, \ldots, x_{n}\right\}$, consequently the probability that each of $k$ points chosen independently and uniformly at random in $K$ falls within conv $\left\{x_{1}, \ldots, x_{n}\right\}$ is given by

$$
\left(\frac{\operatorname{vol} \operatorname{conv}\left\{x_{1}, \ldots, x_{n}\right\}}{\operatorname{vol} K}\right)^{k} \text {. }
$$

Thus, as the points $x_{1}, \ldots, x_{n}$ are distributed independently and uniformly in $K$,

$$
p_{n, k}=\frac{E V_{n}^{k}}{(\operatorname{vol} K)^{k}} .
$$


From (2.1), (2.2), and (2.3) we conclude that

$$
\left(\begin{array}{c}
n+k \\
k
\end{array}\right) \frac{E V_{n}^{k}}{(\operatorname{vol} K)^{k}}=E\left(\begin{array}{c}
n+k-N_{n+k} \\
k
\end{array}\right)
$$

and since

$$
\begin{array}{rl}
\left(\begin{array}{c}
n+k \\
k
\end{array}\right)^{-1} & E\left(\begin{array}{c}
n+k-N_{n+k} \\
k
\end{array}\right) \\
& =E \frac{\left(n+k-N_{n+k}\right)\left(n+k-1-N_{n+k}\right) \cdots\left(n+1-N_{n+k}\right)}{(n+k)(n+k-1) \cdots(n+1)} \\
& =E \prod_{i=1}^{k}\left(1-\frac{N_{n+k}}{n+i}\right),
\end{array}
$$

the first statement of Theorem 1 follows. The second statement is a simple consequence of the first one, since the identity

$$
(1-z)^{k}=\sum_{j=1}^{k}(-1)^{j-1}\left(\begin{array}{l}
k \\
j
\end{array}\right)\left(1-z^{j}\right)
$$

which is easily verified, implies that

$$
\frac{E D_{n}^{k}}{(\operatorname{vol} K)^{k}}=E\left(1-\frac{V_{n}}{\operatorname{vol} K}\right)^{k}=\sum_{j=1}^{k}(-1)^{j-1}\left(\begin{array}{c}
k \\
j
\end{array}\right) E\left(1-\frac{V_{n}^{j}}{(\operatorname{vol} K)^{j}}\right) \text {. }
$$

Remark 1. Observe that neither in the expression for $E V_{n}^{k}$ nor in the expression for $E D_{n}^{k}$ do "mixed" moments of the type $E N_{n+j_{1}}^{i_{1}} N_{n+j_{2}}^{i_{2}}$ with $j_{1} \neq j_{2}$ occur. Analogously to the form of (vol $K)^{-k} E V_{n}^{k}$ stated in the Introduction, (vol $\left.K\right)^{-k} E D_{n}^{k}$ can be written in the form

$$
\begin{aligned}
\left(\begin{array}{l}
k \\
1
\end{array}\right) \frac{1}{n+1} E N_{n+1}+\left(\begin{array}{l}
k \\
2
\end{array}\right)\left[-\left(\frac{1}{n+1}+\frac{1}{n+2}\right) E N_{n+2}+\frac{1}{(n+1)(n+2)} E N_{n+2}^{2}\right] \\
+\cdots+\left(\begin{array}{l}
k \\
k
\end{array}\right)\left[(-1)^{k-1}\left(\frac{1}{n+1}+\cdots+\frac{1}{n+k}\right) E N_{n+k}\right. \\
\left.+\cdots+\frac{1}{(n+1) \cdots(n+k)} E N_{n+k}^{k}\right] .
\end{aligned}
$$

Remark 2. If the uniform distribution on $K$ is replaced by some other probability distribution in $\mathbb{R}^{d}$, the reasoning leading to (2.1) and (2.2) still works as long as single points do not have positive measure. In the argument yielding (2.3), the ratio of the volume of the convex hull of $n$ random points to the volume of $K$ then has to be replaced by the probability content of the convex hull of $n$ random points. The resulting identity relates moments of the probability content of the convex hull of $n$ random points to moments of the number of vertices of the convex hull of $n+k$ random points. 
Remark 3. Efron [17, p. 335] considered the random variable $U_{n}=n-N_{n}$ and derived that

$$
E U_{n}^{k}=\sum_{r=1}^{k} N(n, k, r) \frac{E V_{n-r}^{r}}{(\operatorname{vol} K)^{r}},
$$

where $N(n, k, r)$ is the number of $k$-tuples from $1,2, \ldots, n$ having exactly $r$ different entries,

$$
N(n, k, r)=\left(\begin{array}{l}
n \\
r
\end{array}\right) \sum_{s=1}^{r}(-1)^{r-s}\left(\begin{array}{l}
r \\
s
\end{array}\right) s^{k} .
$$

The case $k=1$ yields

$$
E U_{n}=n \frac{E V_{n-1}}{\operatorname{vol} K}
$$

whence, replacing $n$ by $n+1$, (1.6) and (1.7) follow immediately. The case $k=2$ is considered separately in Remark 5 below. In the general case the right-hand side of (2.5) involves moments of $k$ different random variables, and Efron did not take the equation into further consideration. Replacing the equation by the system

$$
E U_{n}^{i}=\sum_{r=1}^{i} N(n, i, r) \frac{E V_{n-r}^{r}}{(\operatorname{vol} K)^{r}} \quad(i=1, \ldots, k)
$$

and using the additional equations to eliminate the occurring moments of the random variables $V_{n-1}, \ldots, V_{n-k+1}$, the remaining moment $E V_{n-k}^{k}$ can be expressed by the moments $E U_{n}^{i}(i=1, \ldots, k)$ and consequently by the moments $E N_{n}^{i}(i=1, \ldots, k)$. Thus $E V_{n}^{k}$ can be expressed by the moments $E N_{n+k}^{i}(i=1, \ldots, k)$. The details, however, appear to be cumbersome and tedious, and the simple structure of formula (1.8) does not become obvious immediately.

\section{The Arising Identity for the Variances and Some Consequences}

Theorem 1 implies an identity for the variances which is almost as simple as Efron's identity (1.6), (1.7) for the expected values:

Corollary 1. Let $K \in \mathcal{K}^{d}, n \in \mathbb{N}$, and $k \in \mathbb{N}$. Then

$$
\frac{\operatorname{var} V_{n}}{(\operatorname{vol} K)^{2}}=\frac{\operatorname{var} D_{n}}{(\operatorname{vol} K)^{2}}=\frac{\operatorname{var} N_{n+2}+d_{n+2}}{(n+1)(n+2)},
$$

where

$$
d_{n}=\left(E N_{n}\right)^{2}-\frac{n}{n-1}\left(E N_{n-1}\right)^{2}-(2 n-1) E N_{n}+2 n E N_{n-1} .
$$

Remark 4. According to Efron's identity (1.6), (1.7), $d_{n}$ can equivalently be expressed by the expected values $E D_{n-1}$ and $E D_{n-2}$ as well as by the expected values $E V_{n-1}$ and $E V_{n-2}$. We note the latter of the resulting formulae,

$$
d_{n}=n^{2}\left(\frac{E V_{n-1}}{\operatorname{vol} K}\right)^{2}-n(n-1)\left(\frac{E V_{n-2}}{\operatorname{vol} K}\right)^{2}-n \frac{E V_{n-1}}{\operatorname{vol} K} .
$$


Remark 5 (Continuation of Remark 3). The case $k=2$ of (2.5) yields

$$
E U_{n}^{2}=n(n-1) \frac{E V_{n-2}^{2}}{(\operatorname{vol} K)^{2}}+n \frac{E V_{n-1}}{\operatorname{vol} K},
$$

and consequently (see the expression for $E U_{n}$ in Remark 3)

$$
\operatorname{var} U_{n}=n(n-1) \frac{E V_{n-2}^{2}}{(\operatorname{vol} K)^{2}}+n \frac{E V_{n-1}}{\operatorname{vol} K}-n^{2}\left(\frac{E V_{n-1}}{\operatorname{vol} K}\right)^{2} .
$$

Apparently, neither Efron, who mentioned this relation in passing [17, p. 335, formula (3.6)], nor subsequent authors paid further attention to it. Taking into account that $\operatorname{var} U_{n}=\operatorname{var} N_{n},(3.2)$ can be rewritten as

$$
\operatorname{var} N_{n}=n(n-1)\left(\frac{\operatorname{var} V_{n-2}}{(\operatorname{vol} K)^{2}}+\left(\frac{E V_{n-2}}{\operatorname{vol} K}\right)^{2}\right)+n \frac{E V_{n-1}}{\operatorname{vol} K}-n^{2}\left(\frac{E V_{n-1}}{\operatorname{vol} K}\right)^{2},
$$

and, applying (3.1), as

$$
\operatorname{var} N_{n}=n(n-1) \frac{\operatorname{var} V_{n-2}}{(\operatorname{vol} K)^{2}}-d_{n} .
$$

Replacing $n$ by $n+2$ just gives Corollary 1 .

As for the result by Cabo and Groeneboom displayed as (1.3), Hüsler [24, p. 111] had already pointed out that it seemed to be incorrect. In fact, if the random variable $D_{n}$ is asymptotically normally distributed with asymptotic mean $E D_{n}$ and asymptotic variance $\operatorname{var} D_{n}$, in order to avoid $D_{n}$ attaining negative values with positive probability, $E D_{n} \geq \eta \sqrt{\operatorname{var} D_{n}}$ has to be fulfilled asymptotically for every $\eta>0$. Otherwise, if there were an $\eta_{0}>0$ such that asymptotically $E D_{n}<\eta_{0} \sqrt{\operatorname{var} D_{n}}$, the probability that

$$
\frac{D_{n}-E D_{n}}{\sqrt{\operatorname{var} D_{n}}} \leq-\eta_{0}
$$

would tend to $\Phi\left(-\eta_{0}\right)=1-\Phi\left(\eta_{0}\right)>0$ (where $\Phi$ is the distribution function of the standard normal distribution), implying that

$$
D_{n} \leq E D_{n}-\eta_{0} \sqrt{\operatorname{var} D_{n}}<0
$$

with positive probability. Since the relation $E D_{n} \geq \eta \sqrt{\operatorname{var} D_{n}}$ can only be fulfilled asymptotically for every $\eta>0$ if the order of $\operatorname{var} D_{n}$ is less than the order of $\left(E D_{n}\right)^{2}$ and since $E D_{n} \sim \frac{2}{3} r n^{-1} \log n$ as $K$ is a convex polygon with $r$ vertices, the order of $\operatorname{var} D_{n}$ has to be less than $n^{-2} \log ^{2} n$. The denominator in (1.3), which exhibits the order of $\operatorname{var} D_{n}$ to be $n^{-1} \log n$, is incompatible with this requirement.

To see what accounts for the discrepancy, recall that the method by Cabo and Groeneboom is based on the approximation of the process of vertices of the convex hull of a uniform sample by the process of extreme points of a realization of a Poisson point process in such a way that the "left-lower boundary" of the convex hull of a uniform sample of size $n$ from the interior of the square $[0, \sqrt{n}]^{2}$ is associated with the "left-lower 
boundary" of the convex hull of a realization of a Poisson point process on $\mathbb{R}_{+}^{2}$ with intensity Lebesgue measure. (See in particular Corollary 2.2 in [18] and the preceding comment at the bottom of p. 334.) Accordingly, if Corollary 2.1 or rather Theorem 2.3 in Section 2 of [15] is assumed to be correct and used as the starting point, the reasoning sketched in Section 3 of [15] leads to

$$
\frac{D_{n}-4\left(\frac{2}{3} \log n\right)}{2 \sqrt{\frac{100}{189} \log n}} \stackrel{\mathcal{D}}{\rightarrow} \mathcal{N}(0,1)
$$

in the case that $K$ is the square $[0, \sqrt{n}]^{2}$, i.e. a square with area $n$, hence to

$$
\frac{n(\operatorname{vol} K)^{-1} D_{n}-4\left(\frac{2}{3} \log n\right)}{2 \sqrt{\frac{100}{189} \log n}}=\frac{(\operatorname{vol} K)^{-1} D_{n}-4\left(\frac{2}{3} n^{-1} \log n\right)}{2 \sqrt{\frac{100}{189} n^{-2} \log n}} \stackrel{\mathcal{D}}{\rightarrow} \mathcal{N}(0,1)
$$

in the case that $K$ is a square with area vol $K$, and, more generally, to

$$
\frac{(\operatorname{vol} K)^{-1} D_{n}-\frac{2}{3} r n^{-1} \log n}{\sqrt{\frac{100}{189} r n^{-2} \log n}} \stackrel{\mathcal{D}}{\rightarrow} \mathcal{N}(0,1)
$$

in the case that $K$ is a convex polygon with $r$ vertices and area vol $K$. To be consistent with these conclusions, in Theorem 3.1 in [15] the expression for $c_{n}$ has to be replaced by $\sqrt{\frac{100}{189} n^{-2} \log n}$. Correspondingly, in the main result, Theorem 3.2, stated as (1.3) in the Introduction, the denominator has to be replaced by $\sqrt{\frac{100}{189} k n^{-2} \log n}$. (In the present paper we write $r$ instead of $k$.) Furthermore, in the numerator the "remaining area" $A_{n}$ ( $D_{n}$ in the notation of the present paper) has to be replaced by the ratio of the "remaining area" to the area of the considered convex polygon $\left((\mathrm{vol} K)^{-1} D_{n}\right.$ in the notation of the present paper).

The arising order of the denominator, $n^{-2} \log n$ instead of $n^{-1} \log n$, meets the requirement of being less than $n^{-2} \log ^{2} n$. A further consideration, however, shows that in the denominator in Theorem 3.2 the constant, $\frac{100}{189}$, which is not affected by the above alterations, cannot be correct either: In view of $\operatorname{var} N_{n} \geq 0$, the identity (1.10) implies $(\operatorname{vol} K)^{-2} \operatorname{var} D_{n} \geq(n+1)^{-1}(n+2)^{-1} d_{n+2}$. Since $d_{n} \sim \frac{2}{3} r \log n$ as $K$ is a convex polygon with $r$ vertices, it follows that, asymptotically, ( $\operatorname{vol} K)^{-2} \operatorname{var} D_{n} \geq \frac{2}{3} r n^{-2} \log n$, whereas - according to a conclusion analogous to the ones by Groeneboom [18, p. 328]the denominator in (3.3) exhibits that $(\operatorname{vol} K)^{-2} \operatorname{var} D_{n} \sim \frac{100}{189} r n^{-2} \log n$. Therefore Theorem 2.3 in [15], which was used as the starting point of the above conclusions, cannot be entirely correct, and a further mistake must have crept in somewhere in the fundamental papers [18] and [15]. It would be very interesting to discover this further mistake.

Beyond the fact that $\operatorname{var} N_{n}$ implies $\operatorname{var} D_{n-2}$ and vice versa, Corollary 1 yields estimates of these quantities even if neither the one nor the other is known, provided that precise information about the asymptotic behaviour of $E N_{n}$ or, equivalently, $E D_{n}$ is available, whence the asymptotic behaviour of $d_{n}$ can be determined. Such precise information was recently obtained by Reitzner for convex bodies with sufficiently smooth boundaries. The following particular case of Reitzner's results (Theorem 1 of [36]; for $d=2$ : Theorem 3 of [34]) suffices for our purpose: 
Let $K \in \mathcal{K}^{d}$ have a boundary of differentiability class $C^{d+6}$ (if $d=2, C^{6}$ is sufficient) with positive Gaussian curvature $\kappa$. Then

$$
\frac{E D_{n}}{\operatorname{vol} K}=c_{2}(K) n^{-2 /(d+1)}+\cdots+c_{d+3}(K) n^{-(d+3) /(d+1)}+O\left(n^{-(d+4) /(d+1)}\right)
$$

as $n \rightarrow \infty$, where the constants satisfy $c_{2 m+1}(K)=0$ for $m \leq d / 2$ if $d$ is even, and $c_{2 m+1}(K)=0$ for all $m$ if $d$ is odd.

An immediate consequence, obtained according to $(n+1)^{-\alpha}=n^{-\alpha}-\alpha n^{-\alpha-1}+$ $O\left(n^{-\alpha-2}\right)$ as $n \rightarrow \infty$, is the remarkable relation

$$
E D_{n}-E D_{n+1}=\frac{2}{d+1} E D_{n} n^{-1}\left(1+O\left(n^{-1 /(d+1)}\right)\right)
$$

as $n \rightarrow \infty$ (which can presumably be established under less restrictive differentiability assumptions).

In order to evaluate $d_{n}$, we write (3.1) in the form

$$
\begin{aligned}
d_{n}= & n(n-1)\left(\frac{E D_{n-2}}{\operatorname{vol} K}-\frac{E D_{n-1}}{\operatorname{vol} K}\right)\left(2-\frac{E D_{n-2}}{\operatorname{vol} K}-\frac{E D_{n-1}}{\operatorname{vol} K}\right) \\
& -n \frac{E D_{n-1}}{\operatorname{vol} K}\left(1-\frac{E D_{n-1}}{\operatorname{vol} K}\right)
\end{aligned}
$$

and conclude from (3.4) and (3.5) that

$$
d_{n}=\frac{3-d}{d+1} C(K) n^{(d-1) /(d+1)}+O\left(n^{(d-2) /(d+1)}\right)
$$

as $n \rightarrow \infty$, where $C(K)$ is the constant $c_{2}(K)$ of formula (3.4). It is known that

$$
C(K)=\eta(d) A(K)(\operatorname{vol} K)^{-(d-1) /(d+1)} .
$$

Here $A(K)$ is the affine surface area of $K$,

$$
A(K)=\int_{\mathrm{bd} K} \kappa^{1 /(d+1)} d \sigma
$$

and $\eta(d)$ is a constant depending only on the dimension,

$$
\eta(d)=\frac{\Gamma(d+1+2 /(d+1))}{2(1+2 /(d+1)) \Gamma(d+1)}\left(\frac{d+1}{\varrho_{d-1}}\right)^{2 /(d+1)},
$$

$\varrho_{d}$ denoting the volume of the $d$-dimensional unit ball. (For arbitrary $d$ the constant $C(K)$ was first determined by Wieacker [44] in the case that $K$ is a ball, where

$$
A(K)=d \varrho_{d}^{2 /(d+1)}(\operatorname{vol} K)^{(d-1) /(d+1)} .
$$

For $d=2$ the constant $C(K)$ is due to Rényi and Sulanke [37], [38]. In the particular case that $K$ is a circular disk, $C(K)$ equals $2 \pi c_{1}, c_{1}$ being the constant mentioned repeatedly in the Introduction.) 
As $\eta(d)>0, A(K)>0$, and consequently $C(K)>0$, the sign of $d_{n}$ for sufficiently large $n$ corresponds to the sign of $(3-d) /(d+1)$, provided that $(3-d) /(d+1) \neq 0$.

Thus, on the one hand, we have $d_{n}>0$ for sufficiently large $n$ if $d=2$, and it follows from Corollary 1 and $\operatorname{var} N_{n+2} \geq 0$ that

$$
\frac{\operatorname{var} D_{n}}{(\operatorname{vol} K)^{2}} \geq \frac{d_{n+2}}{(n+1)(n+2)},
$$

whence

$$
\operatorname{var} D_{n} \geq \frac{1}{3} \eta(2) A(K)\left(\frac{\operatorname{vol} K}{n}\right)^{5 / 3}+O\left(\frac{1}{n^{2}}\right)
$$

as $n \rightarrow \infty$. Observing that $\eta(2)=\left(\frac{2}{3}\right)^{1 / 3} \Gamma\left(\frac{5}{3}\right)$, our result can be stated in the following form:

Corollary 2. Let $K \in \mathcal{K}^{2}$ have a boundary of differentiability class $C^{6}$ with positive Gaussian curvature $\kappa$. Then

$$
\liminf _{n \rightarrow \infty} \operatorname{var} D_{n}\left(\frac{n}{\operatorname{vol} K}\right)^{5 / 3} \geq \frac{1}{3}\left(\frac{2}{3}\right)^{1 / 3} \Gamma\left(\frac{5}{3}\right) \int_{\mathrm{bd} K} \kappa^{1 / 3} d \sigma .
$$

On the other hand, we have $d_{n}<0$ for sufficiently large $n$ if $d \geq 4$, and it follows from Corollary 1 and $\operatorname{var} D_{n} \geq 0$ that

$$
\operatorname{var} N_{n+2} \geq-d_{n+2},
$$

whence

$$
\operatorname{var} N_{n} \geq \frac{d-3}{d+1} \eta(d) A(K)\left(\frac{n}{\operatorname{vol} K}\right)^{(d-1) /(d+1)}+O\left(n^{(d-2) /(d+1)}\right)
$$

as $n \rightarrow \infty$. Thus we obtain:

Corollary 3. Let $K \in \mathcal{K}^{d}, d \geq 4$, have a boundary of differentiability class $C^{d+6}$ with positive Gaussian curvature $\kappa$. Then

$$
\liminf _{n \rightarrow \infty} \operatorname{var} N_{n}\left(\frac{\operatorname{vol} K}{n}\right)^{(d-1) /(d+1)} \geq \frac{d-3}{d+1} \eta(d) \int_{\mathrm{bd} K} \kappa^{1 /(d+1)} d \sigma,
$$

where $\eta(d)$ is given by (3.7).

Presumably, Corollaries 2 and 3 can be improved and extended as follows:

Conjecture 1. Let $K \in \mathcal{K}^{d}, d \geq 2$, have a sufficiently smooth boundary with positive Gaussian curvature $\kappa$. Then

$$
\lim _{n \rightarrow \infty} \operatorname{var} N_{n}\left(\frac{\operatorname{vol} K}{n}\right)^{(d-1) /(d+1)}=\alpha(d) \int_{\mathrm{bd} K} \kappa^{1 /(d+1)} d \sigma
$$


and

$$
\lim _{n \rightarrow \infty} \operatorname{var} D_{n}\left(\frac{n}{\operatorname{vol} K}\right)^{(d+3) /(d+1)}=\beta(d) \int_{\mathrm{bd} K} \kappa^{1 /(d+1)} d \sigma,
$$

where $\alpha(d)$ and $\beta(d)$ are constants depending only on $d$.

If Conjecture 1 turns out to be true, $\alpha(d)$ and $\beta(d)$ are determined in the case $d=2$ by the constants $c_{1}$ and $c_{2}$ mentioned in the Introduction and in Section 5.3: $\alpha(2)=\pi^{1 / 3} c_{2}$, $\beta(2)=\pi^{1 / 3}\left(\frac{1}{3} c_{1}+c_{2}\right)=\frac{1}{3} \eta(2)+\alpha(2)$. Corollary 1 and (3.6) yield for arbitrary $d$ the relation

$$
\beta(d)=\frac{3-d}{d+1} \eta(d)+\alpha(d) .
$$

Thus $\alpha(d)$ and $\beta(d)$ coincide in the case $d=3$ where $(3-d) /(d+1)$ vanishes. In other words:

Conjecture 2. Let $K \in \mathcal{K}^{3}$ have a sufficiently smooth boundary. Then

$$
\lim _{n \rightarrow \infty} \frac{\operatorname{var} D_{n}}{\operatorname{var} N_{n}}\left(\frac{n}{\operatorname{vol} K}\right)^{2}=1 .
$$

\section{The Distribution of the Number of Vertices}

Theorem 2. Let $K \in \mathcal{K}^{d}$ and $m \in \mathbb{N}$. Then, for $l=1, \ldots, m$, the probability $p_{l}^{(m)}$ that $N_{m}=l$ is given by

$$
p_{l}^{(m)}=(-1)^{l}\left(\begin{array}{c}
m \\
l
\end{array}\right) \sum_{j=1}^{l}(-1)^{j}\left(\begin{array}{l}
l \\
j
\end{array}\right) \frac{E V_{j}^{m-j}}{(\operatorname{vol} K)^{m-j}} .
$$

Proof. Relation (2.4) is equivalent to

$$
\left(\begin{array}{c}
n+k \\
k
\end{array}\right) \frac{E V_{n}^{k}}{(\operatorname{vol} K)^{k}}=\sum_{i=1}^{n+k}\left(\begin{array}{c}
n+k-i \\
k
\end{array}\right) p_{i}^{(n+k)}
$$

Thus we have for $j=1, \ldots, m$ that

$$
\left(\begin{array}{c}
m \\
j
\end{array}\right) \frac{E V_{j}^{m-j}}{(\operatorname{vol} K)^{m-j}}=\sum_{i=1}^{m}\left(\begin{array}{c}
m-i \\
m-j
\end{array}\right) p_{i}^{(m)} .
$$

It is immediately verified that the inverse of the matrix $\left(\left(\begin{array}{c}m-i \\ m-j\end{array}\right)\right)_{i=1, \ldots, m ; j=1, \ldots, m}$ is given by $\left((-1)^{j+l}\left(\begin{array}{c}m-j \\ m-l\end{array}\right)\right)_{j=1, \ldots, m ; l=1, \ldots, m}$. (Observing that both $\left(\begin{array}{c}m-i \\ m-j\end{array}\right)$ and $\left(\begin{array}{c}m-j \\ m-l\end{array}\right)$ are different from zero only if $i \leq j \leq l$ and that

$$
\left(\begin{array}{c}
m-i \\
m-j
\end{array}\right)\left(\begin{array}{l}
m-j \\
m-l
\end{array}\right)=\left(\begin{array}{l}
m-i \\
m-l
\end{array}\right)\left(\begin{array}{l}
l-i \\
l-j
\end{array}\right)
$$


the inner product of any row of the first matrix with any column of the second matrix can be easily evaluated.) Consequently

$$
p_{l}^{(m)}=\sum_{j=1}^{m}(-1)^{j+l}\left(\begin{array}{c}
m-j \\
m-l
\end{array}\right)\left(\begin{array}{c}
m \\
j
\end{array}\right) \frac{E V_{j}^{m-j}}{(\operatorname{vol} K)^{m-j}}
$$

for $l=1, \ldots, m$. Hence Theorem 2 follows as $\left(\begin{array}{c}m-j \\ m-l\end{array}\right)$ is different from zero only if $j \leq l$ and as

$$
\left(\begin{array}{c}
m-j \\
m-l
\end{array}\right)\left(\begin{array}{c}
m \\
j
\end{array}\right)=\left(\begin{array}{l}
m \\
l
\end{array}\right)\left(\begin{array}{l}
l \\
j
\end{array}\right)
$$

Remark 6. If $n<d+1$, the probability that $N_{n+k}$ attains one of the values $n+1, \ldots$, $n+k$ is one. Thus the probability that one of the quantities

$$
1-\frac{N_{n+k}}{n+i} \quad(i=1, \ldots, k)
$$

vanishes is one, and in the first identity of Theorem 1 both sides reduce to zero. (Consequently, in the second identity of Theorem 1 both sides reduce to one.)

Correspondingly, if $m<d+1$, the identity in Theorem 2 reduces to $p_{m}^{(m)}=1$ and $p_{l}^{(m)}=0$ for $l=1, \ldots, m-1$. Furthermore, if $m \geq d+1$, still $p_{l}^{(m)}$ vanishes for $l=1, \ldots, d$, and the identity simplifies to

$$
p_{l}^{(m)}=(-1)^{l}\left(\begin{array}{c}
m \\
l
\end{array}\right) \sum_{j=d+1}^{l}(-1)^{j}\left(\begin{array}{l}
l \\
j
\end{array}\right) \frac{E V_{j}^{m-j}}{(\operatorname{vol} K)^{m-j}}
$$

for $l=d+1, \ldots, m$.

Remark 7. Efron's identity mentioned as (2.5) in Remark 3 can easily be derived from Theorem 2: Express

$$
E U_{n}^{k}=\sum_{l=1}^{n}(n-l)^{k} p_{l}^{(n)}
$$

according to Theorem 2, change the order of summation in the arising double sum, note that

$$
\left(\begin{array}{l}
n \\
l
\end{array}\right)\left(\begin{array}{l}
l \\
j
\end{array}\right)=\left(\begin{array}{c}
n \\
n-j
\end{array}\right)\left(\begin{array}{l}
n-j \\
n-l
\end{array}\right)
$$

replace $n-j$ and $n-l$ by $r$ and $s$, respectively, and recall the definition of $N(n, k, r)$ from Remark 3. It follows that

$$
E U_{n}^{k}=\sum_{r=1}^{n-1} N(n, k, r) \frac{E V_{n-r}^{r}}{(\operatorname{vol} K)^{r}}
$$

This sum is equivalent to the sum in (2.5), since, on the one hand, if $n-1>k$, the summands corresponding to $r=k+1, \ldots, n-1$ vanish as $N(n, k, r)=0$ for $r>k$, and, on the other hand, if $k>n-1$, the summand corresponding to $r=n$ vanishes as then $E V_{n-r}^{r}=0$, while the summands corresponding to $r=n+1, \ldots, k$ vanish as $N(n, k, r)=0$ for $r>n$. 


\section{Concluding Remarks}

\subsection{A Question of Baryshnikov}

It had been proved in an earlier paper [11, Theorem 3] that the probabilities $p_{l}^{(d+3)}(l=$ $d+1, d+2, d+3$ ) can be expressed by the moments $E V_{d+1}$ and $E V_{d+1}^{2}$ :

$$
\begin{aligned}
& p_{d+1}^{(d+3)}=\left(\begin{array}{c}
d+3 \\
2
\end{array}\right) \frac{E V_{d+1}^{2}}{(\operatorname{vol} K)^{2}}, \quad p_{d+2}^{(d+3)}=\left(\begin{array}{c}
d+3 \\
2
\end{array}\right)\left(\frac{E V_{d+1}}{\operatorname{vol} K}-2 \frac{E V_{d+1}^{2}}{(\operatorname{vol} K)^{2}}\right), \\
& p_{d+3}^{(d+3)}=1-\left(\begin{array}{c}
d+3 \\
2
\end{array}\right)\left(\frac{E V_{d+1}}{\operatorname{vol} K}-\frac{E V_{d+1}^{2}}{(\operatorname{vol} K)^{2}}\right) .
\end{aligned}
$$

This result is a particular case of the present Theorem 2 since

$$
E V_{d+2}=\frac{d+2}{2} E V_{d+1}
$$

(Theorem 2 of [11]; see also [12]).

Baryshnikov [6] called the probabilities $p_{d+1}^{(m)}(m=d+2, d+3, \ldots)$ Sylvester numbers. According to the identity mentioned as (1.14) in the Introduction, the probabilities $p_{l}^{(d+3)}$ stated above can equivalently be expressed by the Sylvester numbers $p_{d+1}^{(d+2)}$ and $p_{d+1}^{(d+3)}$ :

$$
p_{d+2}^{(d+3)}=\frac{d+3}{2} p_{d+1}^{(d+2)}-2 p_{d+1}^{(d+3)}, \quad p_{d+3}^{(d+3)}=1-\frac{d+3}{2} p_{d+1}^{(d+2)}+p_{d+1}^{(d+3)} .
$$

In view of this relation Baryshnikov asked (for $d=2$, the dimension, however, does not matter) whether it can be extended from $p_{l}^{(d+3)}(l=d+1, d+2, d+3)$ to $p_{l}^{(m)}(l=d+1, \ldots, m), m>d+3$ : "A really interesting question is if there exists a functional dependence of $p_{l}^{(m)}$ from some finite set of Sylvester numbers."

Theorem 2 shows that an appropriate finite set in order to express the probabilities $p_{l}^{(m)}$ consists of the moments $E V_{m-1}, E V_{m-2}^{2}, \ldots, E V_{d+1}^{m-d-1}$. If $m=d+3$, these moments, i.e. $E V_{d+2}$ and $E V_{d+1}^{2}$, are equivalent to the Sylvester numbers $p_{d+1}^{(d+2)}$ and $p_{d+1}^{(d+3)}$, respectively, as described above. If $m>d+3$, such a relation between the mentioned moments and the Sylvester numbers does not exist. Observe that all Sylvester numbers are known if $K$ is a $d$-dimensional ball, a triangle, or a parallelogram (recall the Introduction), whereas it appears to be difficult even to determine $E V_{m-2}^{2}$ if $K$ is a $d$-dimensional ball and $m>d+3$ or if $K$ is a triangle or a parallelogram and $m>6$. (For $m=6$, see Section 5.2.) If it were in fact possible to express the probabilities $p_{l}^{(m)}$ and hence the moments of $N_{m}$ in terms of Sylvester numbers, according to Theorem 1 the moments $E V_{n}^{k}$ with $n+k=m$ could also be expressed in terms of Sylvester numbers. In other words: The moments $E V_{n}^{k}(n \geq d+1, k \in \mathbb{N})$-and thus in particular $E V_{n}^{2}$ and $\operatorname{var} V_{n}$-could be expressed by the moments $E V_{d+1}^{k}(k \in \mathbb{N})$. 


\subsection{A Consequence of Recent Work by Valtr}

As mentioned in the Introduction, $E V_{n}^{k}$ is not known for any convex body $K$ if $k>1$ and $n>d+1$. Theorem 2 can be used to deduce $E V_{4}^{2}$ if $K$ is a triangle or a parallelogram from recent results by Valtr: The particular case $l=m=6$ of (4.1) yields

$$
\frac{E V_{4}^{2}}{(\operatorname{vol} K)^{2}}=\frac{1}{15} p_{6}^{(6)}+\frac{4}{3} \frac{E V_{3}^{3}}{(\operatorname{vol} K)^{3}}+\frac{2}{5} \frac{E V_{5}}{\operatorname{vol} K}-\frac{1}{15} .
$$

If $K$ is a triangle or a parallelogram, the values of $p_{6}^{(6)}$ are $\frac{91}{900}$ [42] and $\frac{49}{400}$ [41], respectively, the values of $(\mathrm{vol} K)^{-3} E V_{3}^{3}$ are $\frac{31}{9000}$ [2] and $\frac{137}{72000}$ [20], and the values of (vol $K)^{-1} E V_{5}$ are $\frac{43}{180}$ [9] and $\frac{79}{360}$ [9]. Hence we find that the value of (vol $\left.K\right)^{-2} E V_{4}^{2}$ is $\frac{181}{4500}$ if $K$ is a triangle and $\frac{859}{27000}$ if $K$ is a parallelogram.

\subsection{Groeneboom's Constant $c_{2}$ and Hsing's Constant $\sigma^{2}$}

It was pointed out in the Introduction that the constants $c_{2}$ and $\sigma^{2}$ are related by

$$
\sigma^{2}=2 \pi\left(\frac{1}{3} c_{1}+c_{2}\right)(\operatorname{vol} K)^{2} .
$$

The exact value of $c_{1}$ is due to Rényi and Sulanke [37]. The constants $c_{2}$ and $\sigma^{2}$ are known in terms of integrals. Whereas Hsing does not touch the question of how the integral for $\sigma^{2}$ could be evaluated, Groeneboom reduces the integral for $c_{2}$ to two still very complicated double integrals for which he states approximate numerical values, without giving any details of how these have been obtained. (Groeneboom derives the numerical value of $c_{2}$ by combining the results on pp. 361 and 362 of [18], in the course of which apparently the factor 2 in front of the integral in the expression for $\operatorname{var}(N(a))$ on p. 361 has got lost.) It would be interesting to determine the exact value of $c_{2}$ or, equivalently, of $\sigma^{2}$.

\subsection{Just One More Word to Groeneboom's Paper}

As already mentioned in Section 1, Groeneboom [18, pp. 328-329] explains that asymptotic formulae for $\operatorname{var} N_{n}$ can be deduced from the central limit theorems stated as (1.1) and (1.2) in the present paper. Groeneboom writes that "it is not hard to show that certain uniform integrability conditions are satisfied" and that "this analysis has not been carried out to save space". It would still be worthwhile to elaborate on the details.

\section{Acknowledgements}

I am indebted to D. Hug, M. Ludwig, A. V. Nagaev, M. Reitzner, and R. Schneider for helpful discussions and valuable remarks. Furthermore, I express my gratitude to an anonymous referee for helpful comments. 


\section{References}

1. F. Affentranger, Aproximación aleatoria de cuerpos convexos, Publ. Mat. Barc. 36 (1992), 85-109.

2. V. S. Alagar, On the distribution of a random triangle, J. Appl. Probab. 14 (1977), 284-297.

3. I. Bárány, Random polytopes in smooth convex bodies, Mathematika 39 (1992), 81-92.

4. I. Bárány, Sylvester's question: The probability that $n$ points are in convex position, Ann. Probab. 27 (1999), 2020-2034.

5. I. Bárány and C. Buchta, Random polytopes in a convex polytope, independence of shape, and concentration of vertices, Math. Ann. 297 (1993), 467-497.

6. Yu. Baryshnikov, Counting the shape of a drum, Adv. in Appl. Math. 17 (1996), 101-116.

7. Yu. Baryshnikov, Supporting-points processes and some of their applications, Probab. Theory Relat. Fields 117 (2000), 163-182.

8. H. Bräker and T. Hsing, On the area and perimeter of a random convex hull in a bounded set, Probab. Theory Relat. Fields 111 (1998), 517-550.

9. C. Buchta, Zufallspolygone in konvexen Vielecken, J. Reine Angew. Math. 347 (1984), 212-220.

10. C. Buchta, Zufällige Polyeder-Eine Übersicht, in Zahlentheoretische Analysis (E. Hlawka, ed.), pp. 1-13, Lecture Notes in Mathematics, Vol. 1114, Springer-Verlag, Berlin, 1985.

11. C. Buchta, On a conjecture of R. E. Miles about the convex hull of random points, Monatsh. Math. 102 (1986), 91-102.

12. C. Buchta, Distribution-independent properties of the convex hull of random points, J. Theoret. Probab. 3 (1990), 387-393.

13. C. Buchta and M. Reitzner, Equiaffine inner parallel curves of a plane convex body and the convex hulls of randomly chosen points, Probab. Theory Relat. Fields 108 (1997), 385-415.

14. C. Buchta and M. Reitzner, The convex hull of random points in a tetrahedron: Solution of Blaschke's problem and more general results, J. Reine Angew. Math. 536 (2001), 1-29.

15. A. J. Cabo and P. Groeneboom, Limit theorems for functionals of convex hulls, Probab. Theory Relat. Fields 100 (1994), 31-55.

16. L. Devroye, Moment inequalities for random variables in computational geometry, Computing 30 (1983), 111-119.

17. B. Efron, The convex hull of a random set of points, Biometrika 52 (1965), 331-343.

18. P. Groeneboom, Limit theorems for convex hulls, Probab. Theory Relat. Fields 79 (1988), $327-$ 368.

19. P. M. Gruber, Comparisons of best and random approximation of convex bodies by polytopes, Rend. Circ. Mat. Palermo (2) Suppl. 50 (1997), 189-216.

20. N. Henze, Random triangles in convex regions, J. Appl. Probab. 20 (1983), 111-125.

21. T. Hsing, On the asymptotic distribution of the area outside a random convex hull in a disk, Ann. Appl. Probab. 4 (1994), 478-493.

22. I. Hueter, The convex hull of a normal sample, Adv. in Appl. Probab. 26 (1994), 855-875.

23. I. Hueter, Limit theorems for the convex hull of random points in higher dimensions, Trans. Amer. Math. Soc. 351 (1999), 4337-4363.

24. J. Hüsler, On the convex hull of dependent random vectors, Rend. Circ. Mat. Palermo (2) Suppl. 41 (1996), 109-117.

25. N. P. Jewell and J. P. Romano, Evaluating inclusion functionals for random convex hulls, Z. Wahrsch. Verw. Gebiete 68 (1985), 415-424.

26. K.-H. Küfer, On the approximation of a ball by random polytopes, Adv. in Appl. Probab. 26 (1994), 876-892.

27. D. Mannion, The volume of a tetrahedron whose vertices are chosen at random in the interior of a parent tetrahedron, Adv. in Appl. Probab. 26 (1994), 577-596.

28. B. Massé, Invariance principle for the deviation between the probability content and the interior point proportion of a random convex hull, J. Appl. Probab. 32 (1995), 1041-1047.

29. B. Massé, On the variance of the number of extreme points of a random convex hull, Statist. Probab. Lett. 44 (1999), 123-130.

30. B. Massé, On the LLN for the number of vertices of a random convex hull, Adv. in Appl. Probab. (SGSA) 32 (2000), 675-681.

31. R. E. Miles, Isotropic random simplices, Adv. in Appl. Probab. 3 (1971), 353-382. 
32. A. V. Nagaev and I. M. Khamdamov, Limit theorems for functionals of random convex hulls, preprint (in Russian), Institute of Mathematics, Academy of Sciences of Uzbekistan, Tashkent, 1991.

33. W. J. Reed, Random points in a simplex, Pacific J. Math. 54(2) (1974), 183-198.

34. M. Reitzner, The floating body and the equiaffine inner parallel curve of a plane convex body, Geom. Dedicata 84 (2001), 151-167.

35. M. Reitzner, Random polytopes and the Efron-Stein jackknife inequality, Ann. Probab. 31 (2003), 21362166.

36. M. Reitzner, Stochastical approximation of smooth convex bodies, Mathematika, in press.

37. A. Rényi and R. Sulanke, Über die konvexe Hülle von $n$ zufällig gewählten Punkten, Z. Wahrsch. Verw. Gebiete 2 (1963), 75-84.

38. A. Rényi and R. Sulanke, Über die konvexe Hülle von $n$ zufällig gewählten Punkten. II, Z. Wahrsch. Verw. Gebiete 3 (1964), 138-147.

39. R. Schneider, Random approximation of convex sets, J. Microscopy 151 (1988), 211-227.

40. R. Schneider, Discrete aspects of stochastic geometry, in Handbook of Discrete and Computational Geometry (J. E. Goodman and J. O’Rourke, eds.), pp. 167-184, CRC Press, Boca Raton, Florida, 1997.

41. P. Valtr, Probability that $n$ random points are in convex position, Discrete Comput. Geom. 13 (1995), 637-643.

42. $\mathrm{P}$. Valtr, The probability that $n$ random points in a triangle are in convex position, Combinatorica $\mathbf{1 6}$ (1996), 567-573.

43. W. Weil and J. A. Wieacker, Stochastic geometry, in Handbook of Convex Geometry (P. M. Gruber and J. M. Wills, eds.), vol. B, pp. 1391-1438, North-Holland/Elsevier, Amsterdam, 1993.

44. J. A. Wieacker, Einige Probleme der polyedrischen Approximation, Diplomarbeit, Albert-LudwigsUniversität, Freiburg im Breisgau, 1978.

Received December 22, 2002, and in revised form March 16, 2004. Online publication July 23, 2004. 